\title{
TU/e emonownen

\section{Automated blinds with light feedback to increase occupant satisfaction and energy saving}

\section{Citation for published version (APA):}

Meerbeek, B. W., de Bakker, C., de Kort, Y. A. W., van Loenen, E. J., \& Bergman, T. (2016). Automated blinds with light feedback to increase occupant satisfaction and energy saving. Building and Environment, 103, 70-85. https://doi.org/10.1016/j.buildenv.2016.04.002

\section{Document license: \\ TAVERNE}

DOI:

10.1016/j.buildenv.2016.04.002

Document status and date:

Published: 13/04/2016

\section{Document Version:}

Publisher's PDF, also known as Version of Record (includes final page, issue and volume numbers)

\section{Please check the document version of this publication:}

- A submitted manuscript is the version of the article upon submission and before peer-review. There can be important differences between the submitted version and the official published version of record. People interested in the research are advised to contact the author for the final version of the publication, or visit the $\mathrm{DOI}$ to the publisher's website.

- The final author version and the galley proof are versions of the publication after peer review.

- The final published version features the final layout of the paper including the volume, issue and page numbers.

Link to publication

\section{General rights}

Copyright and moral rights for the publications made accessible in the public portal are retained by the authors and/or other copyright owners and it is a condition of accessing publications that users recognise and abide by the legal requirements associated with these rights.

- Users may download and print one copy of any publication from the public portal for the purpose of private study or research.

- You may not further distribute the material or use it for any profit-making activity or commercial gain

- You may freely distribute the URL identifying the publication in the public portal.

If the publication is distributed under the terms of Article 25fa of the Dutch Copyright Act, indicated by the "Taverne" license above, please follow below link for the End User Agreement:

www.tue.nl/taverne

Take down policy

If you believe that this document breaches copyright please contact us at:

openaccess@tue.nl

providing details and we will investigate your claim. 


\title{
Automated blinds with light feedback to increase occupant satisfaction and energy saving
}

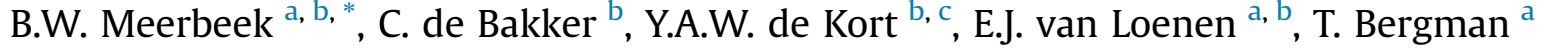 \\ a Philips Lighting Research, High Tech Campus 7, 5656 AE Eindhoven, The Netherlands \\ b Intelligent Lighting Institute, Eindhoven University of Technology, P.O. Box 513, 5600 MB Eindhoven, The Netherlands \\ ${ }^{\mathrm{c}}$ Human Technology Interaction, Eindhoven University of Technology, P.O. Box 513, 5600 MB Eindhoven, The Netherlands
}

\section{A R T I C L E I N F O}

\section{Article history:}

Received 12 January 2016

Received in revised form

29 March 2016

Accepted 4 April 2016

Available online 6 April 2016

\section{Keywords:}

Building automation

User control

Daylight

Blinds

Technology acceptance

User interface

Virtual window

\begin{abstract}
A B S T R A C T
With the increase of building automation in the work environment, there is a risk that occupants lose their sense of control when decisions on environmental aspects such as temperature, electric lighting, and daylight are made by technology. This paper reports two experiments in which we investigated the effect of the level automation and the type of system expressiveness on users' satisfaction with an automated blinds system installed on a virtual window. An expressive interface was designed to communicate the status and intentions of the blinds system to the building occupants. The results show that the addition of the expressive interface increased user satisfaction compared to the original system. Moreover, users made less corrections after automatic blind adjustments and adherence to the system suggestions increased. These results demonstrate the potential of expressive interfaces to increase user's acceptance of automated blinds and thereby realizing the anticipated energy savings.
\end{abstract}

() 2016 Elsevier Ltd. All rights reserved.

\section{Introduction}

\subsection{Perceived control in automated office buildings}

The increasing attention for energy efficient buildings combined with technological advances in sensors, processing power, lighting, and networks drive the development of so called 'Smart Buildings'. Simple forms of building intelligence such as occupancy sensing or daylight-based dimming are already common practice. There are clear economical drivers for building automation. For example, energy and cost savings can be realized by automatically switching off the light when people are not in a room or by dimming the electric light if sufficient daylight is available. Building automation should not only result in energy and cost savings, but also make sure that occupants are satisfied with and feel in control of their working environment. User acceptance is essential for successful adoption of building automation technologies, but at the same time difficult to achieve. A balance between energy efficiency and occupant comfort needs to be found, ensuring that people feel

\footnotetext{
* Corresponding author. Philips Lighting Research, High Tech Campus 7, 5656 AE Eindhoven, The Netherlands.

E-mail address: bernt.meerbeek@philips.com (B.W. Meerbeek).
}

comfortable and productive at their workplace while preserving the energy saving potential of building automation technologies.

Both in the domain of technology acceptance and in the domain of the built environment, a sense of control is generally recognized as an important factor influencing comfort and satisfaction (e.g. $[2,4,13])$. Perceived control is included in technology acceptance models and user satisfaction measures (e.g. [31]). Veitch [32] describes perception of control as an important psychological process that influences perceived lighting quality and satisfaction with the working environment. In her study, people with dimming control reported higher ratings of lighting quality, environmental satisfaction, self-rated productivity, and even showed more sustained motivation and improved performance on a measure of attention. Similarly, Newsham and colleagues [21] showed in a laboratory study that the provision of dimming control for a lighting system resulted in improvements on several factors including mood, satisfaction with the environment, and self-assessed productivity. Lee and Brand [14] have investigated the effect of control over the office workspace on perceptions of the work environment and work outcomes. Based on a questionnaire study among more than 200 office workers, they concluded that having personal control over the physical working environment positively influences both job satisfaction and group cohesiveness. 
With the increase of building automation in the work environment, there is a risk that occupants lose their sense of control when decisions on environmental aspects such as temperature, electric lighting, and daylight are made by technology.

\subsection{Automatic and manual blinds operations}

Previous research on automatic and manual blind systems in the work environment indicates the importance of appropriate daylight control for energy saving and user comfort. Several studies investigated the use of manual blinds and show that people do not regularly change the blinds positions $[11,5,23,26]$. People generally lower the blinds to block direct sunlight, but often forget to retract them. If people retract blinds, they mainly do this to increase daylight entrance or to create a view [6]. Interestingly however, Reinhart and Voss [28] found that in $88 \%$ of the cases when the blinds were lowered automatically, people manually raised them within $15 \mathrm{~min}$, indicating a low acceptance of automatic blind adjustments. Guillemin and Morel [7] developed and evaluated a selfadaptive integrated system for energy and comfort management in buildings, in which the blinds control system was optimized for visual comfort if a user was present and for thermal comfort in absence of a user. Although the solution demonstrated its potential for reducing the energy consumption, the questionnaire results showed that users quickly got angry at the automatic system when it did not take into account their wishes. Vine and colleagues [33] investigated office workers' response to an automated interior venetian blind system with a linked electric lighting system. In a pilot study, 14 participants experienced three modes of operation during sessions of one hour per mode. The three modes varied in degree of control that was available to the user. The general levels of satisfaction were similar among the three modes of operation, although there seemed to be a tendency that in the manual control mode participants were more satisfied with the lighting conditions than in the automatic user control mode. Participants seemed to be least satisfied with the automatic mode. However, the sample size and time frame of the study, as well as the differences found, are too small to make conclusive statements about the effect of control mode on satisfaction with the lighting. Sadeghi and colleagues [29] performed a comparative study on occupant interactions with shading and lighting systems using four different control interfaces, including a fully automatic system, an automatic system with manual overrides via a remote control, manual control via a wall switch, and manual control via a web interface. The fully automatic system resulted in the lowest scores on comfort. Comfort votes were increased when manual override was possible or when manual control was offered via the web interface or wall switch. They further emphasize the importance of accessibility of the controls. Similarly, Bakker and colleagues [2] showed that having the possibility to manually overrule the automated facade leads to higher user satisfaction with light levels on the work plane and in the room. Based on a literature review, Galasiu and Veitch [6] concluded that photo-controlled lighting systems are most widely accepted when there is individual override control. Integrated control for both lighting and shading can be acceptable, but are most widely accepted when a degree of manual control is provided. Another literature review on dynamically controlled shading systems confirms the importance of simple manual controls for acceptance of automated shading systems [12]. Although these cited studies clearly show the importance of personal control for occupants' comfort, several studies highlighted that occupant control of blinds and lighting can significantly increase energy demand in a building [8,9]. In recent work, Meerbeek and colleagues [20] reported a field study in 40 Dutch offices in which they monitored the blinds usage of an automated blinds system over a period of 20 weeks. The results showed that a majority of the building occupants (77.5\%) switched off the automatic mode of the blinds system permanently. Simulation results indicated that this significantly impacts the energy consumption in the building. The estimated total daily average energy consumption for heating and cooling was significantly lower for occupants using the automatic mode than for manual users [20]. One of the reasons for switching off the automatic mode was that occupants did not understand why the blinds were moving up or down. They felt this was often occurring at the wrong moments.

\subsection{Expressive interface for automated systems}

To help people understand and accept the behaviour of automated systems, appropriate communication from the system towards the users is deemed a crucial factor. This communication might be provided by an expressive interface which provides information to the end-user about the internal reasoning, intentions, and actions of the automated system. In particular when tasks are only partially automated, "it is essential that each party, human and machine, know what the other is doing and what is intended." [22]. Often, expressive interfaces come in a human-like representation as people are wired to communicate with other people and experts in interpreting verbal and non-verbal signals from other human beings. Experiments have shown that people are inclined to attribute human characteristics to technology while interacting with it and to perceive the systems as social actors [27]. It has been found in other domains that people tend to attribute personality traits to automated systems [19]. This attributed system personality might help in the interaction between users and technology as it allows people to form a conceptual model of the system. It channels behaviour, beliefs, and intentions into a cohesive, consistent set of behaviours [22].

Expressive interfaces might also affect users' perception of control. Generally, information, choice and predictability are the three prominent factors that are found to influence perceived level of control [30]. In the context of this study, the expressive interface might provide the user with information related to the blinds status or outside conditions and give the user options to choose from (e.g. lowering or raising the blinds). In addition, the expressive interface might help users to predict the automatic behaviour of the system, for example by signalling an automatic blind change before it is effectuated.

\subsection{Research questions}

In this study we investigate the user satisfaction and actual usage of an automated blinds system with an expressive interface. More specifically, we research the effect of the level automation and the type of system expressiveness on users' satisfaction with and usage of the blinds system installed on a virtual window with LED spot to mimic sunlight. Two experiments are conducted to address this research question. In the first experiment $(\mathrm{N}=48)$, three levels of automation and two types of expressiveness are compared in a controlled mixed design user study in a laboratory setting to find their main effects on user satisfaction and blinds usage as well as the interaction effects between level of automation and type of expressiveness (Section 3). In the second experiment $(\mathrm{N}=24)$, two types of expressiveness with the same level of automation are compared, again through a user study in a laboratory setting, to zoom in on the effects of the type of expressiveness on satisfaction and usage (Section 4). But first, Section 2 describes the design of the expressive interface and the levels of automation that were tested in these two experiments. 


\section{Design of expressive interface for automated blinds}

\subsection{Automated blinds system}

An automated interior venetian blinds systems was developed with automatic behaviour comparable to the system that was evaluated in a previous field study [20]. In order to test in a controlled setting and not depend on actual variations in daylight conditions, an office environment with a virtual window was created in which daylight situations could be simulated [17]. Direct sunlight was mimicked with an LED spotlight and its light output thresholds were set as triggers for the blinds systems to lower or raise the blinds (described in more detail in Section 3 and 4). The designed light conditions served the main purpose of this study to evaluate various expressive interfaces and automation strategies with the blinds in a relatively short period and in a controlled way, without being dependent on or affected by actual variations in real daylight conditions. The virtual window with an 'outside view' stimulated participants to have the blinds open, while the LED spot was able to create glare and stimulated participants to lower the blinds. It should be noted that the light intensity, spectrum, and glare perception thresholds were specific for the virtual window and LED spot configuration of the two studies and therefore not generalizable to real daylight conditions. For example, people generally are more tolerant for glare from real daylight with a view than from artificial light sources.

The automated blinds system was extended with an expressive interface to communicate to the users about its' status and intentions. This feedback mechanism should be 'ambient' and embedded in the building environment informing users in an unobtrusive way. This is considered an important quality for our system, since office workers expect it working quietly in the background without disturbing them, while at the same time being attentive and responsive to their needs. Various examples of ambient information systems have been developed for other domains using displays, sounds, everyday objects, or art pieces to inform the user [25]. In this study, we explore the use of a lighting device embedded in the blinds to communicate to users about its' status and intentions. Light is a medium that can be directed and moved in or outside people's field of view. Therefore, it can easily move from the centre of our attention to the periphery and back again [1].

The ambient light feedback device should provide users with information on the actual outside daylight conditions that trigger automatic blind changes (or in case of the experiments, of the mimicked daylight conditions). Furthermore, the system should provide the user with information about upcoming or recommended blind changes to increase the predictability of the system. The provisioning of information and enhanced predictably are expected to contribute to increased perception of control and higher satisfaction with the automated blind system. Moreover, in an earlier study by Maan and others [15]; ambient light feedback was found to influence users' behaviour. They tested the effect of feedback provided by a lighting device that gradually changed its colour dependent on energy consumption and compared it with the effect of numerical feedback. The ambient light feedback was found to have stronger persuasive effects. Hence, it is expected that the expressive interface not only contributes to a higher perception of control and increased user satisfaction but also affects the actual blinds usage.

We designed two variants of the ambient light feedback (moderately expressive vs highly expressive) and three levels of automation (low vs medium vs high) to evaluate the impact of these design parameters on the user satisfaction and usage of the blinds system.

\subsection{Light feedback}

The light feedback system was designed to be mounted on top of a motorized blind system installed in front of a virtual window. It consists of a pixelated LED strip with individually controllable LEDs. On top of this LED strip, a transparent light guide panel (Evonik EndLighten $\mathrm{TM}$ ) was placed such that the light from the LED strip coupled into the side of this light guide material. The light guide diffuses the light and couples it out sideways such that an evenly illuminated panel is created. Laser cuts were made in the light guide panel and acted as mirrors due to total internal reflection (TIR) of the light, resulting optically in twelve individually addressable light segments. The ten segments in the centre form the blocks that indicate the level of solar radiation, ranging from level 1 (low radiation) to level 10 (high radiation), while the two segments on the sides are shaped as an arrow up and an arrow down to indicate the recommended or effectuated blind change (Fig. 1). Each of these segments can be switched on and off separately by controlling the underlying LEDs independently. In on state, the segment takes the colour of the LEDs that couple the light into it, and in off state the segments become transparent and practically invisible. The behaviour of the light feedback system was programmed with Arduino.

\subsection{Levels of automation}

The level of automation was manipulated on three levels varying in decision and action selection in line with the automation scale of Parasuraman [24]. The high level automation was comparable to the systems as investigated in the field study of Meerbeek and colleagues [20], and can be placed on level 10 on Parasuraman's scale (Table 1) as it does not involve the user in its decision making (although users could overrule its decisions afterwards and have manual control over the blinds). In the medium level of automation, users were able to accept or reject a blinds change suggested by the system (level 6 on Parasuraman's scale). If a user did not act within 50 seconds from the moment a suggestion was made the suggested blinds change would be implemented. The system would approve users' actions if they lowered the blinds to minimally $50 \%$ or raised them to minimally $25 \%$ and in that case would not undertake further action. In the low level of automation, the system would still suggest a blinds change but users were given the full responsibility to implement the suggested blinds change.

\subsection{Types of expressiveness}

Two types of expressiveness were created. Version A ('gradual') expressed its intentions with a gradual colour change of the arrows, starting from green and changing to red to indicate a higher urgency for adjusting the blinds. Fig. 2 shows some examples of the

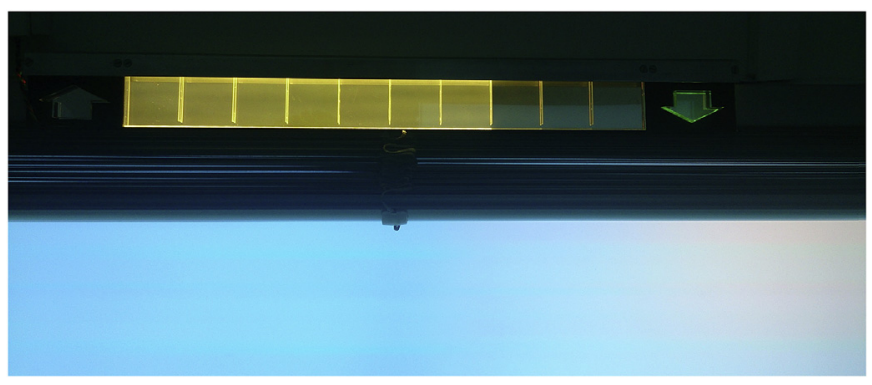

Fig. 1. Light feedback system mounted on top of a motorized blinds system in front of a virtual window. 
Table 1

Mapping of levels of automation in Parasuraman's scale to three automation levels in this study [24].

\begin{tabular}{|c|c|c|}
\hline & Parasuraman's scale & Level of automation in this study \\
\hline 10 & System decides everything, acts autonomously, ignoring the human & High \\
\hline 9 & System informs the human only if system decides to & \\
\hline 8 & System informs the human only if asked & \\
\hline 7 & System executes automatically, then necessarily informs the human & \\
\hline 6 & System allows the human a restricted time to veto before automatic execution & Medium \\
\hline 5 & System executes a suggestion if the human approves & \\
\hline 4 & System suggest one alternative & Low \\
\hline 3 & System narrows the selection down to a few & \\
\hline 2 & System offers a complete set of decision/action alternatives & \\
\hline 1 & System offers no assistance; human must take all decisions and actions & \\
\hline
\end{tabular}

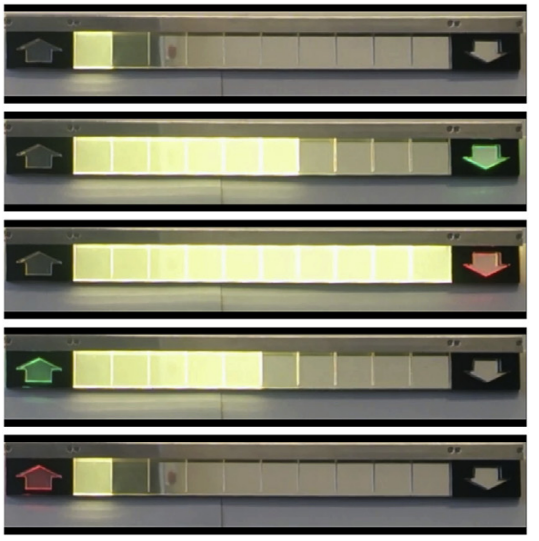

A. Blinds are up, sun level 1

B. Blinds are up, sun level 6: green arrow down to suggest lowering the blinds

C. Blinds are up, sun level 10: red arrow down to suggest lowering the blinds

D. Blinds are down, sun level 5: green arrow up to suggest raising the blinds

E. Blinds are down, sun level 1: red arrow up to suggest raising the blinds

Fig. 2. Example of light feedback in different situations.

light feedback for Version A at various 'sun light' intensities, suggesting the users to adjust the blinds with the coloured arrows. With version B ('pulsating'), the arrows were red and pulsating at increasing rate to indicate an increasing urgency to adjust the blinds. The arrows increased their pulsing rate in three steps. First, the arrows were on for 1 second and off for 1 second, so a pulsing rate of $0.5 \mathrm{~Hz}$. The pulsing rate then increased to successively $1 \mathrm{~Hz}$ and $2 \mathrm{~Hz}$. For the original blinds system (high level of automation) there was no light feedback system.

\section{Experiment 1: evaluation of light feedback with different levels of automation}

\subsection{Study design}

Both level of automation and type of expressiveness were varied on two levels, which resulted in four different versions of the system. We wanted to compare these versions with the original system as evaluated in the field study [20], so that it could be investigated whether the light feedback affected users' behaviour and whether these changes indeed improved users' satisfaction. To reduce the length of the experiment it was decided to test the type of expressiveness between subjects and level of automation within subjects. So each participant experienced three versions in a balanced order: the original blinds system without light feedback, and two levels of automation (low and medium) with the same type of expressiveness. Moreover, participants were randomly assigned to the type of expressiveness. The mixed experiment design resulted in the five conditions ( $\mathrm{C} 0-\mathrm{C} 4)$ presented in Table 2.

Various dependent variables were included in the study design, but in this paper we only report on the variables relevant to the research question posed in the introduction of this paper: perceived system personality, perceived control, user satisfaction, and user behaviour. Other variables, including for example perceived ease of use, perceived usefulness, user characteristics, and trust in the system are outside the scope of this paper.

\subsection{Materials and setting}

The experiment was conducted in the ExperienceLab of Philips Research, which resembled an office setting with a virtual window. An abstract view was created at this virtual window with which we want to give participants the idea of a real view to outside, so they would be motivated to raise the blinds to create an 'outside' view [17]. The lower part of the window was rendered green to prompt the idea of a meadow and the upper part blue to suggest the idea of a sky. The ceiling lighting fixtures (4000K LED) in the space were set to provide a horizontal illuminance of 300 lux at the desk, which is the minimum for tasks like writing, typing, reading and data processing in the office according to building regulations (NEN-EN 12464). A spotlight was used to induce the impression of a sun. At each window, a motorized blinds system (Somfy LW 25 E83 with mat grey-silver coloured slats) was installed covering the full width of the virtual window. Both blinds were controlled simultaneously and could be operated by the experimenter pc (Pharos Designer timelines for automatic blind adjustments) and by the participant via a web interface on a tablet computer. Fig. 3 and Fig. 4 gives an impression of the virtual window, spotlight, and blinds in experiment 1.

A light scenario was created to mimic a sun breaking through the clouds and then after a while disappearing behind the clouds again to create situations in which the blinds need to be adjusted. As one of the main reason for users to manually lower the blinds is the prevention of discomfort glare [20], we wanted the system to 
Table 2

Overview of conditions experiment 1 .

\begin{tabular}{|c|c|c|c|}
\hline & No expressive feedback (none) & Version A (gradual) & Version B (pulsating) \\
\hline Low automation & & $\begin{array}{l}C 1\left(A_{\text {low, }} E_{\text {grad }}\right) \\
\text { System only suggest; Green-to-red light }\end{array}$ & $\begin{array}{l}C 3\left(A_{\text {low, }}, E_{\text {puls }}\right) \\
\text { System only suggest; Pulsating red light }\end{array}$ \\
\hline Medium automation & & $\begin{array}{l}\mathrm{C} 2\left(\mathrm{~A}_{\text {med, }} \mathrm{E}_{\text {grad }}\right) \\
\text { System suggests and acts; Green-to-red light }\end{array}$ & $\begin{array}{l}C 4\left(A_{\text {med, }} E_{\text {puls }}\right) \\
\text { System suggests and acts; Pulsating red light }\end{array}$ \\
\hline High automation & $\begin{array}{l}\mathrm{CO}\left(\mathrm{A}_{\text {high, }}, \mathrm{E}_{\text {none }}\right) \\
\text { Original system }\end{array}$ & & \\
\hline
\end{tabular}

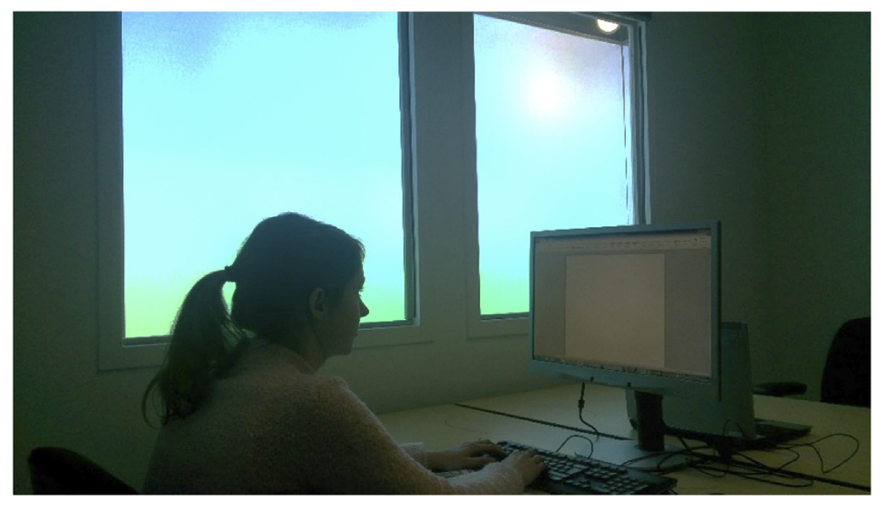

Fig. 3. Setting experiment 1.

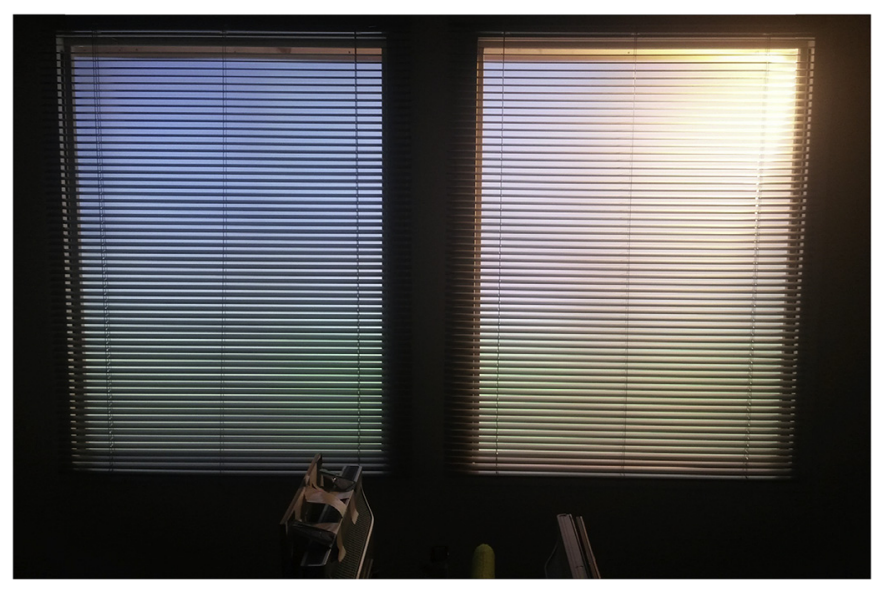

Fig. 4. Virtual window in experiment 1 with blinds lowered and with horizontal slats. suggest users to lower the blinds before users actually experienced discomfort glare. This would be interesting to investigate, as users were prompted to perform an action while they did not yet feel uncomfortable but that action would be preferred from an energy saving perspective. As the commonly acceptable methods and guidelines for calculating discomfort glare from daylight or electric lighting are not validated for daylight mimicking systems such as virtual windows and existing metrics do not always correlate well to subjective glare perceptions [16], the thresholds for discomfort glare were set based on a few trials with lighting experts evaluating level of discomfort at various light intensities of the sun mimicking spot. The threshold for discomfort glare was consequently set at a vertical illuminance $\left(E_{v}\right)$ of 950 lux at the eye of the participant. The intensity of the spotlight was raised linearly in ten steps from a base level of 470 lux $\left(E_{v-\min }\right)$ to its maximum of 1230 lux $\left(E_{v-\max }\right)$, measured at eye height of the participant when seated and also included the lighting provided by the virtual window and the ceiling lights. It should be stressed that these threshold values were determined by subjective evaluations of discomfort glare by a group of lighting experts. These evaluations were not only subjective, but also specific to the experimental setting (participants viewpoint, virtual window, LED spot light, etc.). Therefore, these values cannot be generalized to other real or virtual daylight situations. However, given the main purpose of our study - which is to evaluate the expressive interfaces and automation strategies and not to optimize an automated blind control algorithm - the subjective method was preferred over an objective glare metric. With the chosen thresholds, we were confident that we could create a discomfort glare situation that would trigger participants to use the blinds.

The light scenario was introduced twice per condition, so that the user could experience the system behaviour twice, and at different moments, so he would not be able to anticipate on its behaviour. An example test procedure for one condition with two light scenarios is visualized in Fig. 5, where the vertical illumination

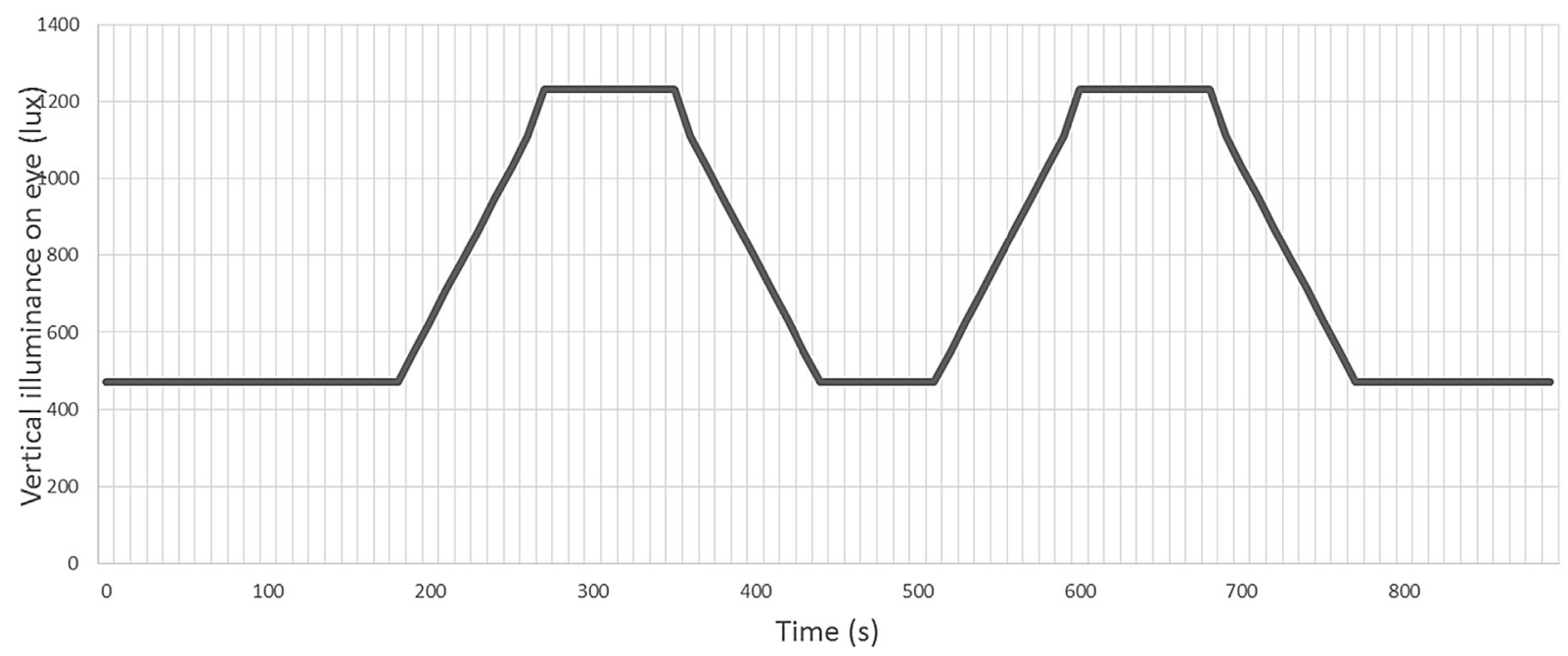

Fig. 5. Light scenario of sun-mimicking LED spot light. 


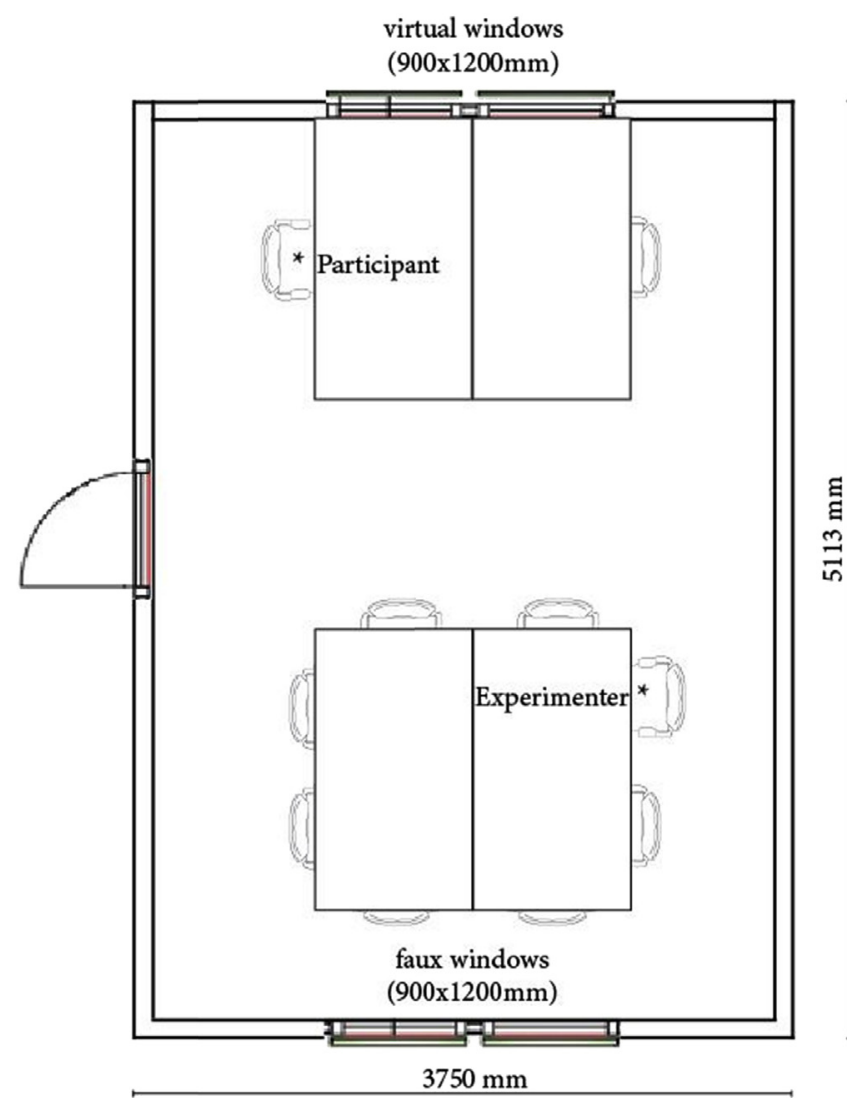

Fig. 6. Layout experiment 1 .

at eye level is plotted over time.

The office setting was created by placing two desks perpendicular to the virtual window (see Fig. 6). In the back of the lab a group of four more desks was placed. The participant was seated at the desk close to the virtual window that was opposite to the spot light, so that the 'sunlight' was able to create discomfort glare. Participants were able to manually operate the blinds via a tablet. They could adjust the position $(0 \%, 25 \%, 50 \%, 75 \%$, and $100 \%)$ and the angle of the blinds (horizontal, vertical, $45^{\circ}$ ) by touching a representative icon on the user interface.

\subsection{Procedure and measurements}

Participants were asked to install themselves behind the desk and to read and sign a consent form. They were provided with a short explanation sheet about the purpose and set-up of the experiment and with a sheet which explained the behaviour of the light feedback system (Appendix A). Each of the three conditions was tested for $15 \mathrm{~min}$. After the testing of each version, the user was asked to fill in a questionnaire which assessed the dependent variables (Appendix B). After the testing of all three versions, a semistructured interview was held to gain a deeper understanding about which aspects of the system participants did and did not like. Users were also asked which version they preferred and why. The total experiment duration was around 90 min per participant.

Perceived system personality was measured with the short form Big Five personality questionnaire [3], with four items per personality dimension, rated on a 7-point scale. Perceived control was assessed with a measure developed for evaluating humancomputer interaction [10]. User satisfaction was measured with an own developed questionnaire with a 7-point Likert scale consisting of the four questions on satisfaction with the overall blinds system, the light feedback system, the automatic blinds behaviour, and with controlling the blinds system via the tablet.

User interaction with the system was logged to evaluate the effect of the tested conditions on user behaviour, in particular the adherence of the user to the suggestions of the system and his corrections of system actions. Adherence was used as a measure to compare users' behaviour on a low and medium level of automation. With a low level of automation, users could only adhere to and not correct the system, as the system did not effectuate any blind adjustment automatically. Adherence (A) was calculated by counting for each condition the times that a user (i) adheres to a system suggestions (a) divided by the total of 4 received suggestions ( 2 suggestions to raise the blinds, 2 suggestions to lower the blinds), averaged over participants ( $\mathrm{n}$ ), and expressed as a percentage (see Equation (1)). The criterion for adherence to a suggestion to raise the blinds was that the blinds were adjusted by the user before the minimum radiation level was reached (level 1), or by the system, with no subsequent blind adjustments (i.e. corrections) from the user until the next sun break through. A similar criterion was used for adherence to suggestions to lower the blinds, but then for radiation levels of 6 or higher.

Users' corrections were defined as actions of users that were a reaction to the system's action and involved a user initiated adjustment of the blinds position (blind angle adjustments were not included as corrections since the automated behaviour only changed the blinds position and not the angle). Correction (C) was calculated by counting for each condition the times a user (i) corrected a system action (c), divided by the total amount of system

Table 4

User satisfaction for the tested conditions ( $\mathrm{A}=$ level of automation, $\mathrm{E}=$ type of expressiveness, $\mathrm{EMM}=$ estimated marginal means, $\mathrm{SE}=$ standard error $)$.

\begin{tabular}{lllll}
\hline Condition & EMM & SE & Mean difference with C0 & p-value \\
\hline CO $\left(A_{\text {high, }} E_{\text {none }}\right)$ & 3.488 & 0.150 & n/a & n/a \\
C1 $\left(A_{\text {low, }} E_{\text {grad }}\right)$ & 4.176 & 0.194 & -0.688 & 0.004 \\
C2 $\left(A_{\text {med, }} E_{\text {grad }}\right)$ & 3.958 & 0.200 & -0.470 & 0.183 \\
C3 $\left(A_{\text {low, }} E_{\text {puls }}\right)$ & 4.190 & 0.211 & -0.701 & 0.010 \\
C4 $\left(A_{\text {med, }} E_{\text {puls }}\right)$ & 4.090 & 0.192 & -0.602 & 0.018 \\
\hline
\end{tabular}

Table 3

Internal consistency (Cronbach's alpha) for scales used in experiment 1.

\begin{tabular}{llc}
\hline Scale & Items & Cronbach's $\alpha$ \\
\hline Extraversion (discarded) & 4 & 0.101 \\
Agreeableness (discarded) & 4 & 0.554 \\
Conscientiousness (discarded) & 4 & 0.369 \\
Neuroticism (discarded) & 4 & 0.517 \\
Openness to new experiences (discarded) & 4 & 0.214 \\
Extraversion \& Openness (talkative, energetic, creative, curious) & 4 & 0.823 \\
Agreeableness \& Emotional Stability (cooperative, polite, calm, relaxed) & 0.863 \\
Perceived control & 2 & \\
Satisfaction & 0.813 \\
\hline
\end{tabular}


actions (s), averaged over participants (n), and expressed as a percentage (see Equation (2)).

$A=\frac{\sum_{i}^{n a_{i}}}{n} \times 100 \%$

Equation (1) Adherence

$C=\frac{\sum_{i}^{n} \frac{c_{i, n}}{s_{i, n}}}{n} \times 100 \%$

Equation (2) Corrections

\subsection{Participants}

Participants were either students or Philips employees. The students were recruited by distributing flyers at the Eindhoven University of Technology, by word-of-mouth and by social media. They received a gift voucher for their participation. Philips employees were mostly interns and did not receive an incentive because they participated during work time. In total, 48 participants took part in the experiment (20 females and 28 males; age range $19-51$, mean $=26.1, \mathrm{SD}=7.2$ ).

\subsection{Questionnaire results}

For each scale, Cronbach's alpha was calculated to test the internal consistency of the measure. The results are presented in Table 3. The internal consistency for the five personality dimensions was low. For conscientiousness, no combination of items resulted in a reliable scale, so this scale had to be discarded. For the other four personality scales, some items were discarded to reach acceptable internal consistency. Given the high correlations between Extraversion and Openness to new experiences on the one hand, and Agreeableness and Neuroticism on the other hand (further named Emotional stability, commonly used if the scale is reversed), these scales were combined for further analysis.

\subsubsection{Perceived system personality}

Perceived system personality was expected to be influenced by both level of automation and type of expressiveness. However, no significant main effect of type of expressiveness on perceived system personality was found. The level of automation did have significant main effects on both Extraversion \& Openness $(F=9.281$, $\mathrm{p}=0.004)$ and Emotional stability \& Agreeableness $(\mathrm{F}=7.578$, $\mathrm{p}=0.008$ ). Participants perceived the system with a low level of automation as less 'extravert and open' than the medium level of automation (2.7 vs 3.1), but more 'emotionally stable and agreeable' (4.2 vs. 3.6).

\subsubsection{Perceived control}

Perceived control was also expected to be influenced by both level of automation and type of expressiveness. However, no significant main effect of type of expressiveness on perceived control was found. Level of automation did have a significant main effect on perceived level of control $(\mathrm{F}=23.859, \mathrm{p}=0.000)$. Participants perceived more control with the low level of automation than with the medium level of automation (5.1 vs 4.0). For both the low and medium level of automation, the perceived level of control was significantly higher than with the original system, with a high level of automation (3.3).

\subsubsection{User satisfaction}

All versions of the system with light feedback (C1-C4) scored higher on user satisfaction than the system without light feedback (CO). Table 4 shows the estimated marginal means for each condition and the results of a paired comparison between the original system ( $\mathrm{CO}$ ) and the other conditions. The two systems with low level of automation (C1 and C3) scored highest on user satisfaction and also the medium level of automation with expressiveness version B (C4) scored significantly higher on user satisfaction than the original system (CO).

\subsection{Usage behaviour}

The number of corrections users made was compared for the three conditions in which an automatic blind adjustment was possible (C0, C2, C4). In case of the original blinds system (C0), on average $50.8 \%(\mathrm{SE}=5.7)$ of the automatic system actions were corrected by the user. For the two versions with light feedback and a medium level automation with expressiveness version $A(C 2)$ and version $\mathrm{B}(\mathrm{C} 4)$, respectively $24.8 \%(\mathrm{SE}=8.4)$ and $31.3 \%(\mathrm{SE}=8.2)$ of system actions were corrected. The difference between $\mathrm{C} 2$ and $\mathrm{C} 0$ was found to be significant $(\mathrm{p}=0.036)$. The results on users' adherence in experiment 1 will be presented in Section 4.7 and compared with experiment 2 .

\subsection{Interview results}

During the interview, participants expressed their preferences with respect to the three versions they had experienced (see Table 5). Due to the mixed design, conditions $\mathrm{C} 1-\mathrm{C} 4$ were each experienced by 24 participants and CO was experienced by all 48 participants. The results show that most participants (87.5\%) preferred the versions with the light feedback device over the original blinds system. There was no clear difference in preference between the low and medium level of automation. The main motivations for their preferences are listed in Table 5 , with in between brackets the number of participants that provided this reason.

Table 5

Users' preferred conditions including main motivations ( $\mathrm{A}=$ level of automation, $\mathrm{E}=$ type of expressiveness).

\begin{tabular}{|c|c|c|}
\hline Condition & Preferred by & Main motivations for preference \\
\hline $\mathrm{CO}\left(\mathrm{A}_{\text {high, }} \mathrm{E}_{\text {none }}\right)$ & 3 out of 24 & $\begin{array}{l}\text { Don't want to put effort in regulating blinds ( } 2 \text { ) } \\
\text { Want to concentrate on my work.(1) }\end{array}$ \\
\hline $\mathrm{C} 1\left(\mathrm{~A}_{\text {low }}, \mathrm{E}_{\text {grad }}\right)$ & 10 out of 24 & $\begin{array}{l}\text { Automatic actions of other versions are conflicting with my preferences (4) } \\
\text { Want to be in control. ( } 3 \text { ) } \\
\text { Don't want to be distracted by automatic behaviour (3) }\end{array}$ \\
\hline $\mathrm{C} 2\left(\mathrm{~A}_{\text {med, }} \mathrm{E}_{\text {grad }}\right)$ & 11 out of 24 & $\begin{array}{l}\text { Want some control myself ( } 3 \text { ) } \\
\text { Like to be informed ( } 3 \text { ) }\end{array}$ \\
\hline $\mathrm{CO}\left(\mathrm{A}_{\text {high, }} \mathrm{E}_{\text {none }}\right)$ & 3 out of 24 & $\begin{array}{l}\text { Don't want to put effort in regulating blinds (2) } \\
\text { Want to concentrate on my work (1). }\end{array}$ \\
\hline C3 $\left(\mathrm{A}_{\text {low }}, \mathrm{E}_{\text {puls }}\right)$ & 10 out of 24 & $\begin{array}{l}\text { Automatic actions of other versions are conflicting with my preferences (5) } \\
\text { Want to be in control. (2) }\end{array}$ \\
\hline$C 4\left(A_{\text {med, }}, E_{\text {puls }}\right)$ & 11 out of 24 & $\begin{array}{l}\text { Don't want to take care of blinds continuously ( } 7 \text { ) } \\
\text { Want some control myself ( } 2 \text { ) }\end{array}$ \\
\hline
\end{tabular}


All transcribed interviews were analysed to reveal the important themes that participants brought up when asked about their experience with the automated blinds system. These themes are convenience, subtlety, fine-grained control, learning, decision support, personal comfort, and transparency. Next, participants' comments on each of these themes are described in more detail, again with in between brackets the number of participants that mentioned it.

Many users indicated that regulating the blinds in the office is of minor importance as they want to focus on their main tasks and working activities. Operating the blinds should cost as little effort as possible (26) and should not require thoughtful consideration (18). Participants liked the fact they could control the blinds from their chairs via the tablet. "I find it too much effort to stand up from my chair and walk to the knob". Many participants particularly liked the fact that the blinds would raise automatically after a period of high radiation (11). "I really like that the blinds go up automatically. It was really a happy moment that more daylight entered the room. Otherwise, it could be that you are busy and in a dark room for an hour."

As most users consider controlling the blinds of secondary importance in their work environment, they want the systems to be subtle in its behaviour and presence. The blinds system should limit the times it interrupts users from their work (25), the feedback system should not be in the centre of attention (12), and the system should be reserved (7). Especially participants that experienced the expressive version with pulsating red light (version B) commented on this aspect. "I think the flickering is a bit too much. If all systems would ask that much attention...".

Although operating the blinds was considered of limited importance, many participants expressed the desire for more finegrained control. For some users, the predefined settings with a choice for a few blinds positions and a few angles was not sufficient, and they want to control the blinds more precisely (18) at a position or angle.

Participants mentioned the importance of a system that adapts to individual preferences, and wanted the system to act automatically according to the users preferences (11) and learn from users' behaviour (11). "I would like to have the system act to my preferences. I don't want to adjust the whole time; that is annoying". "The system should actually learn what appropriate behaviour is". Some participants also mentioned they want a way to communicate their preferences to the system (4).

Many participants liked the light feedback and mention the system should support users in making a decision (23). "I think the mentioned they want the system to communicate its actions to the user in advance (7) so they can anticipate. "When it is automatic, I don't know when it is going to move. But when I change them myself then I can take a water or something until the noise is over".

\section{Experiment 2: effect of type of light feedback on satisfaction and usage}

\subsection{Introduction}

The results in experiment 1 showed no significant effects of the type of expressiveness on perceived system personality and perceived control. This factor was tested between subjects, while level of automation - which had significant effects on perceived system personality and perceived control - was tested within subjects. It could be that the effects of expressiveness were not found due to this experiment design. Therefore, a second experiment was designed to test the type of expressiveness within subjects at a fixed level of automation. Furthermore, the measure for perceived system personality was adapted given the low internal consistency in experiment 1.

\subsection{Study design}

The design of experiment 2 resembles to a large extent the design of the first experiment. In this section, we highlight the differences between the study designs. In experiment 2, the level of automation was held constant at the medium level, as this allowed to investigate the mixed control situation including the number of system actions corrected by the user (see Table 6). Two types of expressiveness were included similar to experiment 1 . However, the behaviour of the arrows was slightly adjusted based on the comments of users in study 1 on the visibility and clarity of the behaviour. Version A in study 1 changed the colour of the arrows from green to red in 10 steps, each displayed for 5 seconds. In study 2 , the colour change consisted of only 3 steps, each displayed for 25 seconds. For version $\mathrm{B}$, the difference in pulsing rate between the start and the end of the light behaviour was increased to make the difference more noticeable to users. In 3 steps, the pulsing rate increased from $0.44 \mathrm{~Hz}$, to $1.33 \mathrm{~Hz}$, to $4 \mathrm{~Hz}$. Furthermore, the light feedback device was now placed under the window instead of above such that it would be easier for users to look at the feedback device. Each participant experienced in a balanced order both expressive versions of the blinds system with a medium level of automation.

Table 6

Overview of conditions experiment 2 ( $\mathrm{A}=$ level of automation, $\mathrm{E}=$ type of expressiveness).

\begin{tabular}{|c|c|c|}
\hline & Version A (gradual) & Version B (pulsating) \\
\hline Medium automation & $\begin{array}{l}\mathrm{C} 2 \mathrm{a}\left(\mathrm{A}_{\text {med, }} \mathrm{E}_{\text {grad }}\right) \\
\text { System suggests and acts; Green-to-red light. }\end{array}$ & $\begin{array}{l}C 4 a\left(A_{\text {med, }}, E_{\text {puls }}\right) \\
\text { System suggests and acts; Pulsating red light. }\end{array}$ \\
\hline
\end{tabular}

feedback is useful, it feels like you get advice". However, another group of participants believes that users should be able to regulate the blinds according to their own comfort (22). "I didn't care about the system's suggestions. I don't care about energy efficiency of the office, it is just about whether I think it is comfortable". "For my comfort, it is not necessary that the system does anything. I experience myself whether it is annoying".

Finally, several participants commented on the lack of transparency of current automated blind systems. They expressed that the system should give users insights into the outside situation, especially when the blinds are lowered and closed (11). Some also

\subsection{Materials and setting}

The experiment was conducted in a usability lab at the Technical University of Eindhoven with two spaces connected by a glass window. In the first space, an office setting was created resembling the office setting in study 1 , while in the second space a virtual natural view (projection) and virtual sun (LED spot) were positioned close to the window to mimic daylight conditions in the first space (see Fig. 7). A light scenario was created comparable to the scenario in study 1 . Due to the differences in experimental setting (room size, window size, reflectance of walls, ceilings, floor, virtual 


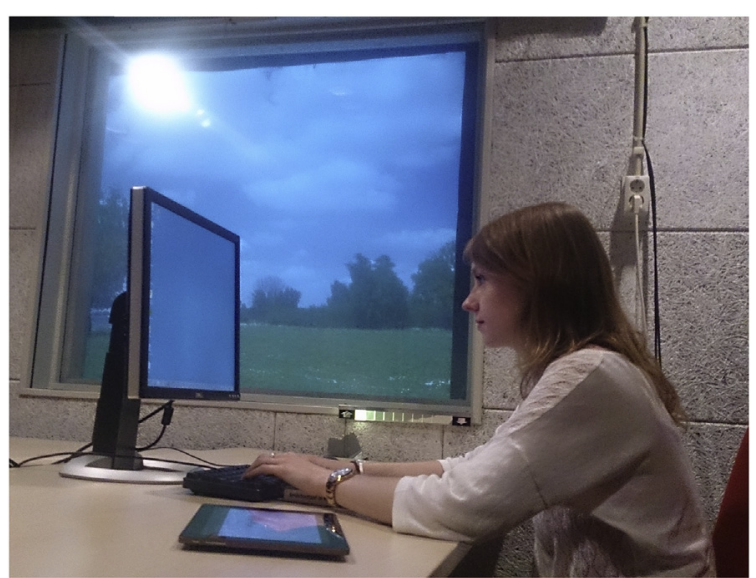

Fig. 7. Experimental setting study 2.

view, etc.), the light intensities of the virtual sun were adapted. Again, the threshold for discomfort glare was set based on a few trials with lighting experts subjectively evaluating level of discomfort at various light intensities of the virtual sun. The same tablet was used to manually control the blinds position and angle.

\subsection{Procedure and measurements}

Also the procedure of experiment 2 was comparable to experiment 1 . However, in study 2 , each participant only experienced two versions. Each version was experienced for 17.5 minutes and included two light scenarios. The total duration per participant was around 70 minutes. After each version, a questionnaire was completed and the experiment ended with a short semi-structured interview, this time focusing on the expressive behaviour of the light feedback device.

Similar measures were used as in experiment 1 but a few measures were adapted (Appendix C). The measure for perceived system personality (based on [3]) was extended with additional personality items selected from [18]. Each personality dimension was measured with 6 items, resulting in a total of 30 items (see Table 7). For each dimension internal consistency was calculated using Cronbach's alpha. A few items had to be dropped to result in scales with moderate to high internal consistency. For satisfaction, two items were discarded to reach an acceptable internal consistency. Satisfaction with the tablet and the automatic behaviour were left out. The remaining items were satisfaction with the overall blinds system and satisfaction with the light feedback system.

\subsection{Participants}

Participants were recruited at the university and all were students or PhD students. They received a gift voucher for their participation. In total, 24 participants took part in this experiment ( 11 females and 13 males; age range $18-30$, mean $=23.2, \mathrm{SD}=3.6$ ).

\subsection{Questionnaire results}

\subsubsection{Perceived system personality}

Perceived system personality was expected to be influenced by the type of expressiveness of the blinds system. Table 8 shows the estimated marginal means for the pulsating (C4a) and gradual (C2a) feedback and the results of a paired comparison. From the data, it can be concluded that C4a was perceived as more extravert, less agreeable, more neurotic, and less open to new experiences than $\mathrm{C} 2 \mathrm{a}$.

\subsubsection{Perceived control}

The type of expressiveness was also expected to influence the level of control perceived by the participants. The results indeed showed a significant $(\mathrm{p}=0.038$ ) difference between the two versions, with C4a resulting in a lower perceived level control $(\mathrm{EMM}=4.0)$ than $\mathrm{C} 2 \mathrm{a}(\mathrm{EMM}=4.5)$.

\subsubsection{User satisfaction}

The user satisfaction levels between C4a (EMM = 3.9) and C2a $(\mathrm{EMM}=4.1)$ were not significantly different (see Table 9).

\subsection{Usage behaviour}

For the gradual (C2a) and pulsating (C4) version of expressive feedback, respectively $3.6 \%(\mathrm{SE}=6.0)$ and $11.6 \%$ ( $\mathrm{SE}=6.1$ ) of system actions were corrected. So participants seemed to correct the system with the gradual feedback (green to red light) less than the system with the red pulsating light, although this difference was not significant $(\mathrm{p}=0.179$ ). The percentages of corrections in study 2 were lower than in study 1 (C2: $24.8 \%$ and C4: $31.3 \%$ ).

Table 10 shows the percentages of participants that adhered to the suggestions of the system in study 1 and study 2 . A user action was counted as adherent if it would coincide with a suggestion made by the system. For lowering blinds this was at radiation level 6 to 10 , while for raising blinds this was at radiation level 5 to 1 . The results show that in general, adherence in study 2 was higher than in study 1 , which can be explained by the fact that the light feedback device was placed at a more visible location under the virtual window. In particular for the lowering of the blinds, more users adhered to the system suggestion in the second study than in the first study.

\section{Discussion and conclusion}

Building automation in the work environment might result in occupants losing their sense of control when decisions on environmental aspects are made by technology. In two experiments, we investigated the user satisfaction and actual usage of an automated blinds system with an expressive interface installed on a virtual

Table 7

Internal consistency (Cronbach's alpha) for scales used in experiment 2.

\begin{tabular}{lll}
\hline Scale & Items & Cronbach's $\alpha$ \\
\hline Extraversion (talkative, assertive, expressive, confident, dominant) & 5 & 0.595 \\
Agreeableness (cooperative, polite, friendly, helpful, agreeable, bossy) & 6 & 5 \\
Conscientiousness (reliable, persistent, firm, consistent, indecisive) & 4 & 0.824 \\
Neuroticism (calm, relaxed, unemotional, patient) & 4 & 0.618 \\
Openness to new experiences (creative, curious, intelligent, analytical) & 2 & 0.613 \\
Perceived control & 0.615 \\
Satisfaction & 0.662 \\
\hline
\end{tabular}


Table 8

Perceived system personality for the two types of expressiveness (EMM = estimated marginal means, SE $=$ standard error).

\begin{tabular}{|c|c|c|c|c|c|c|}
\hline & \multicolumn{2}{|c|}{ C4a (pulsating) } & \multicolumn{2}{|c|}{ C2a (gradual) } & \multicolumn{2}{|c|}{ Paired comparison } \\
\hline & EMM & SE & EMM & SE & EMM difference & $\mathrm{p}$ \\
\hline Extraversion & 3.885 & 0.182 & 3.427 & 0.182 & 0.458 & 0.025 \\
\hline Agreeableness & 3.521 & 0.174 & 4.063 & 0.174 & -0.542 & 0.008 \\
\hline Conscientiousness & 4.058 & 0.174 & 4.169 & 0.174 & -0.110 & 0.337 \\
\hline Emotional stability & 3.292 & 0.174 & 3.875 & 0.174 & -0.583 & 0.009 \\
\hline Openness to new experiences & 2.979 & 0.131 & 3.188 & 0.131 & -0.208 & 0.050 \\
\hline
\end{tabular}

window that was designed to enhance users' perception of control and increase system acceptance. More specifically, the effect of the level automation and the type of system expressiveness on users' satisfaction with and usage of the blinds was explored.

The first experiment showed that the level of automation influences the perceived system personality. Participants perceived the system with a low level of automation as less 'extravert and open' than the system with a medium level of automation, but more 'emotionally stable and agreeable'. Furthermore, as expected, participants perceived more control with the low level of automation than with the medium level of automation. For both low and medium level of automation, the perceived level of control was significantly higher than with the original system, with a high level of automation. The results also showed that the expressive feedback of the light feedback device increased user satisfaction compared to the original system. The feedback also impacted users' blinds usage and reduced the number of system actions that users corrected.

While the results of study 1 did not show any significant effects of the type of expressiveness, the results of the second study showed that the type of expressiveness (when tested within subjects) did affect the perceived system personality. The version with pulsating light feedback was perceived as more extravert, less agreeable, less emotionally stable, and less open to new experiences than the version with gradual light feedback. Furthermore, the pulsating feedback resulted in a lower perceived level control than the gradual feedback. Study 2 also showed the effect of the type of expressiveness on user behaviour. The pulsating feedback seemed to result in a slightly higher adherence to system suggestions than the gradual feedback. Overall, the adherence in study 2 was higher than in study 1 , which can be explained by the fact that the light feedback device was placed at a more visible location. Finally, with the original system in study $1,50.8 \%$ of the system actions was corrected by user. In the second study, with the gradual light feedback, the percentage of corrections was only $3.6 \%$. This indicates that the expressive interface, by providing the light feedback, increased the user acceptance of automatic blind

Table 9

Estimated Marginal Means for User satisfaction of C4a and C2a (EMM = estimated marginal means, $\mathrm{SE}=$ standard error).

\begin{tabular}{|c|c|c|c|c|c|c|}
\hline & \multicolumn{2}{|c|}{$\begin{array}{l}\text { C4a } \\
\text { (pulsating) }\end{array}$} & \multicolumn{2}{|c|}{ C2a (gradual) } & \multicolumn{2}{|c|}{ Paired comparison } \\
\hline & EMM & SE & EMM & SE & EMM difference & $\mathrm{p}$ \\
\hline User satisfaction & 3.875 & 0.215 & 4.125 & 0.215 & -0.250 & 0.349 \\
\hline
\end{tabular}

Table 10

Percentage of participants adhering to the suggestions of the blinds system.

\begin{tabular}{|c|c|c|c|c|}
\hline & \multicolumn{2}{|c|}{$\begin{array}{l}\text { Type of expressiveness (study } \\
\text { 1) }\end{array}$} & \multicolumn{2}{|c|}{ Type of expressiveness (study 2 ) } \\
\hline & C2 (gradual) & C4 (pulsating) & C2a (gradual) & C4a (pulsating) \\
\hline Lowering & $38.6 \%$ & $29.6 \%$ & $77.5 \%$ & $75.0 \%$ \\
\hline Raising & $87.5 \%$ & $72.7 \%$ & $87.5 \%$ & $90.0 \%$ \\
\hline
\end{tabular}

adjustments substantially.

There are a few limitations to the studies presented in this work. First, the experiments were conducted in a controlled setting, evaluating only the initial user experience with the blinds system. Daylight and view were simulated. It requires further research to validate the findings in a real office environment under real daylight conditions and over a longer period. For example, during the experiments participants experienced a "breakthrough of the sun' twice within 15 minutes, which is more frequent than on a typical day and might have resulted in more negative perception of the more red pulsating feedback. With further validation in a realistic setting, the impact of the expressive interface on blinds usage and hence energy usage can be more accurately predicted. Furthermore, many individual differences were observed between user preferences for type of expressiveness and level of automation. In future work, the role of individual user characteristics in preference for automated blinds systems should be further investigated. Third, we only evaluated one form of expressive interface for automated blinds, namely a light strip. However, many more expressive interfaces could be designed to achieve a similar effect. It would also be interesting to compare the effectiveness of different implementations of expressive interfaces. For example, the 'personality' of the expressive interface could be adapted to suit the preferences of individual users. Finally, our studies and results are limited to closed office settings and single users. Additional research is needed to investigate user satisfaction with automated blinds and the role of expressive interfaces in open plan offices, as many other factors are expected to play a role, including for example social dynamics.

Despite the limitations, the two studies show many promising results and interesting findings that to our knowledge have not been reported before. This study confirmed previous findings in other domains that people tend to attribute a personality to automated systems for an automated system in the built environment. Not only the level of automation, but also the way a system communicates with the user affects the perceived system personality and how much control users perceive while interacting with the system. The results further show how these factors can impact user's satisfaction with the automated system and the way these systems will be used. The increased adherence to system suggestions and the large reduction in the number of corrections made by the user clearly indicates the potential of expressive interface to increase user's acceptance of automated blinds and thereby realizing the anticipated energy savings. Therefore, expressive interfaces might be instrumental for the future success of building automation systems and ensuring that these will be embraced by occupants and create energy efficient and comfortable work environments.

\section{Acknowledgements}

This research was performed within the framework of the strategic joint research program on Intelligent Lighting between TU/e and Koninklijke Philips N.V. 


\section{Appendix A. Participant instructions}

\section{Version C1}

When the strength of the sun is too high the right arrow will turn green to indicate that the blinds need to go down.

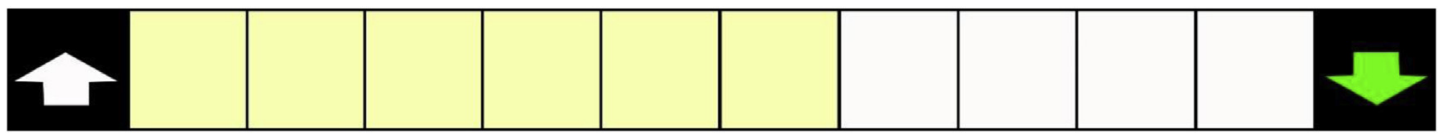

This is however only a suggestion, you will need to set them yourself to the position at which you want them to be. If you lower the blinds the arrow will turn off.

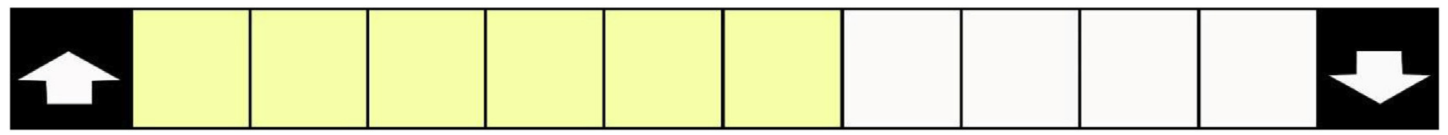

If you do not lower the blinds while the strength of the sun increases the arrow will gradually turn into red.
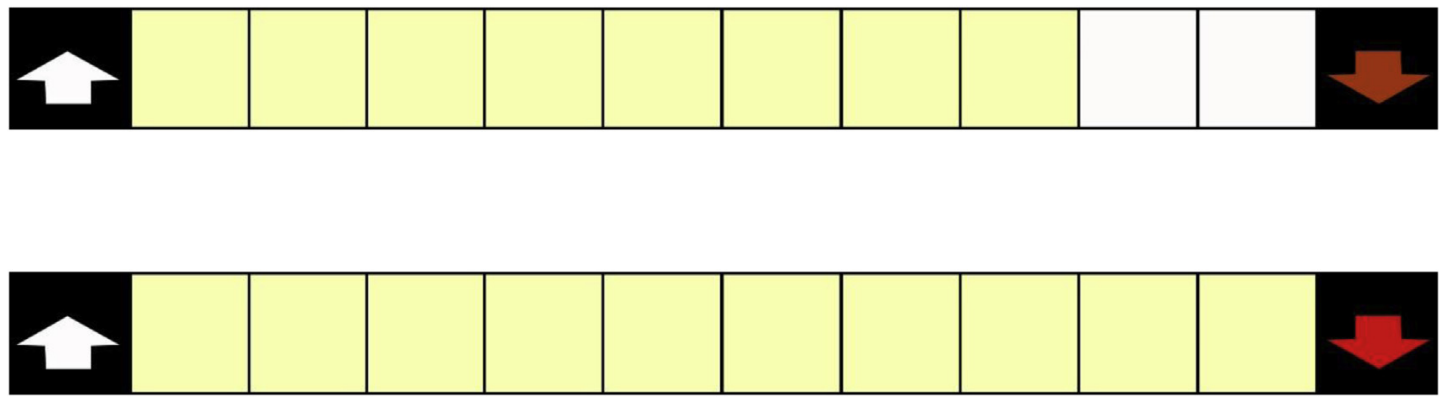

When the sun then loses strength again the left arrow will turn green to indicate that the blinds need to go up.

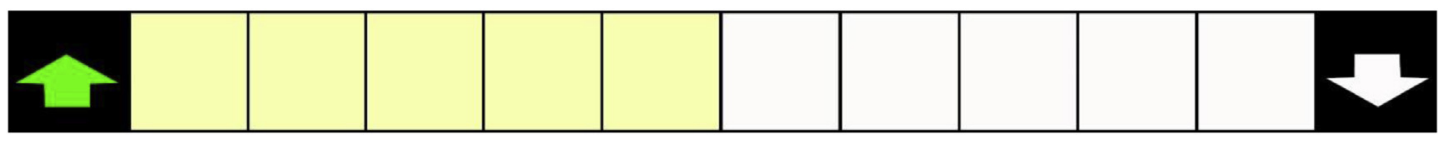

This is however only a suggestion, you will need to set them yourself to the position you want them.

If you raise the blinds the arrow will turn off.

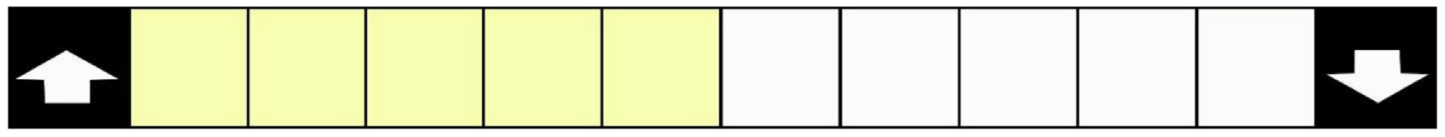

If you do not raise the blinds while the strength of the sun decreases the arrow will gradually turn into red.
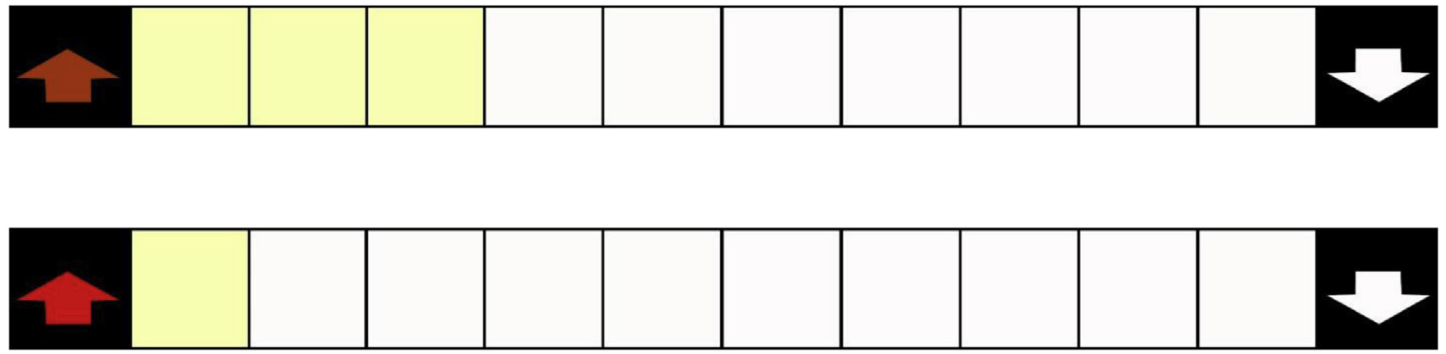
Version $C 2$

When the strength of the sun is too high the right arrow will turn green to indicate that the blinds need to go down.

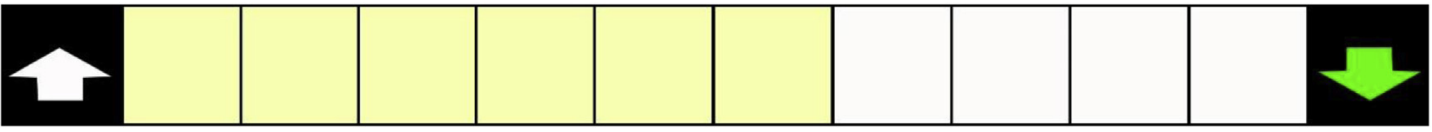

If you lower the blinds the arrow will turn off.

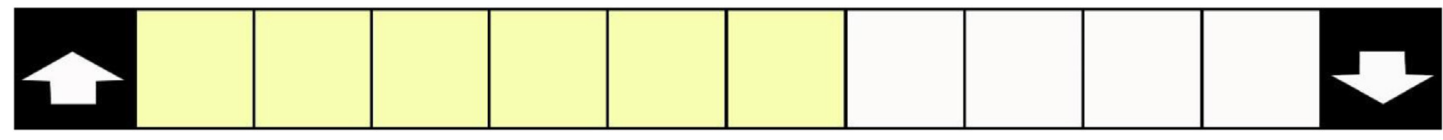

If you do not lower the blinds while the strength of the sun increases the arrow will gradually turn into red.
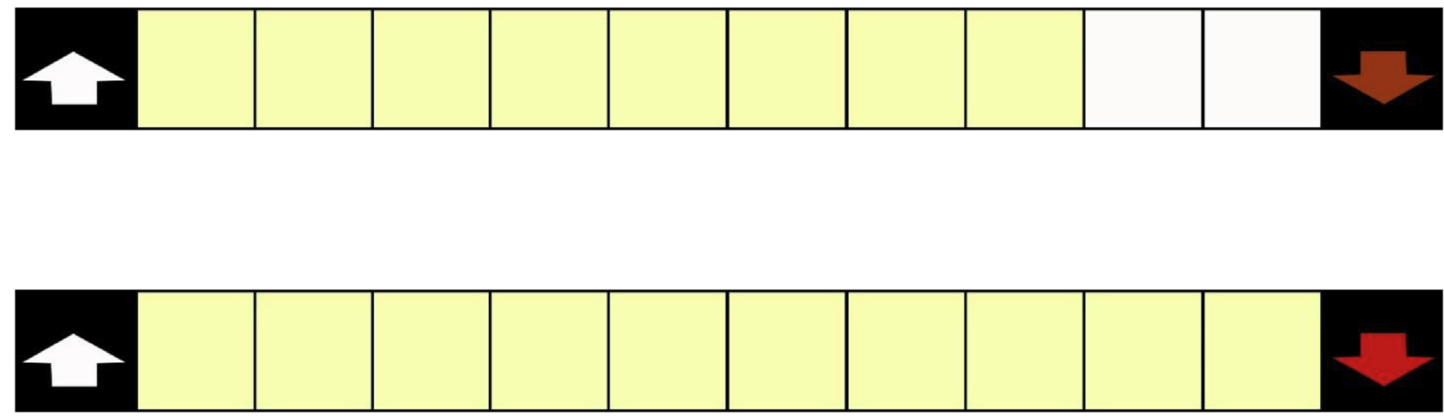

If you then still did not lowered the blinds they will automatically go fully down.

When the sun then loses strength again the left arrow will turn green to indicate that the blinds need to go up.

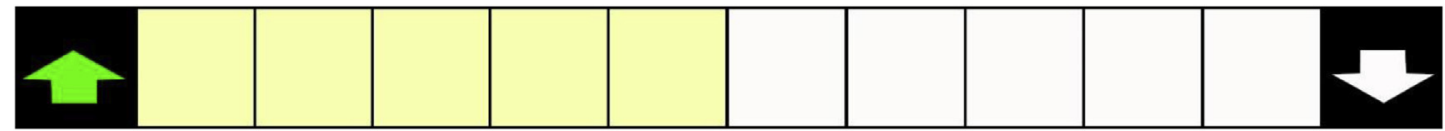

If you raise the blinds the arrow will turn off.

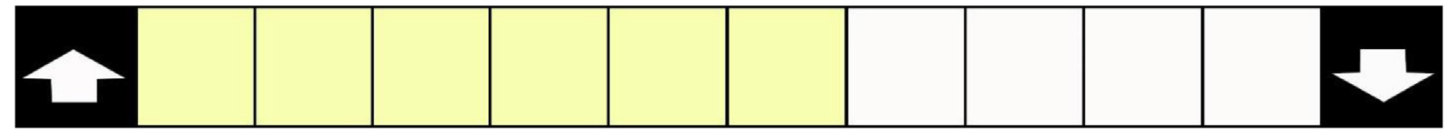

If you do not raise the blinds the arrow while the strength of the sun decreases the arrow will gradually turn into red.
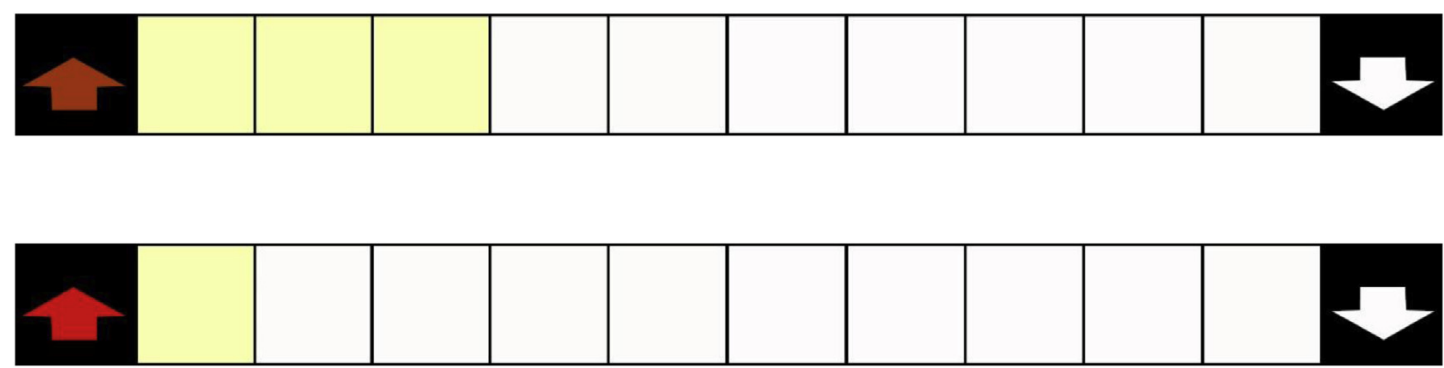
If you then still did not raised the blinds they will automatically go fully down.

\section{Version C3}

When the strength of the sun is too high the right arrow will turn red and flicker to indicate that the blinds need to go down.

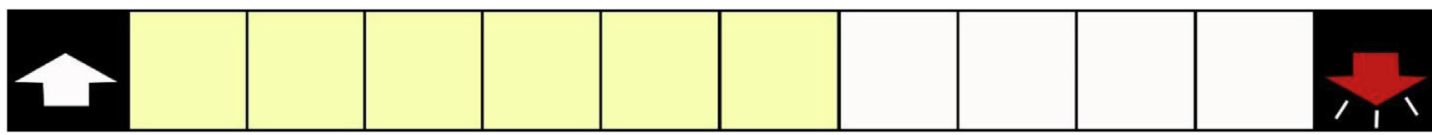

This is however only a suggestion, you will need to set them yourself to the position at which you want them to be. If you lower the blinds the arrow will turn off and stop flickering.

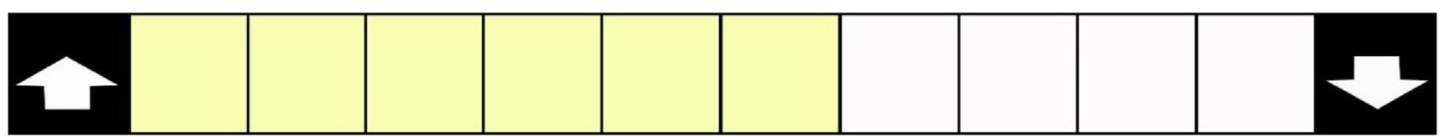

If you do not lower the blinds while the strength of the sun increases the arrow will gradually flicker faster.
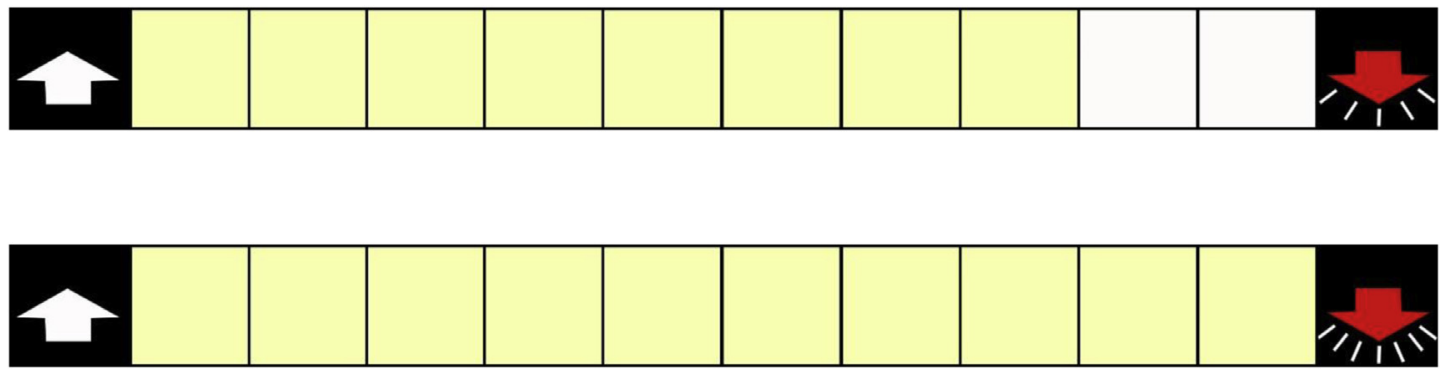

When the sun then loses strength again the left arrow will turn red and flicker to indicate that the blinds need to go up.

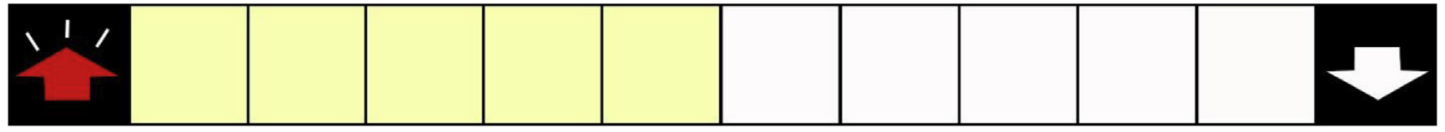

This is however only a suggestion, you will need to set them yourself to the position you want them. If you raise the blinds the arrow will turn off and stop flickering.

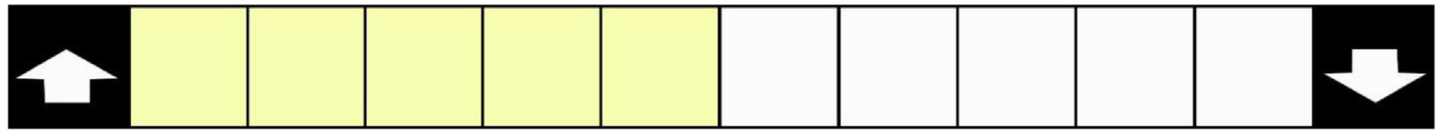

If you do not raise the blinds while the strength of the sun decreases the arrow will gradually flicker faster.
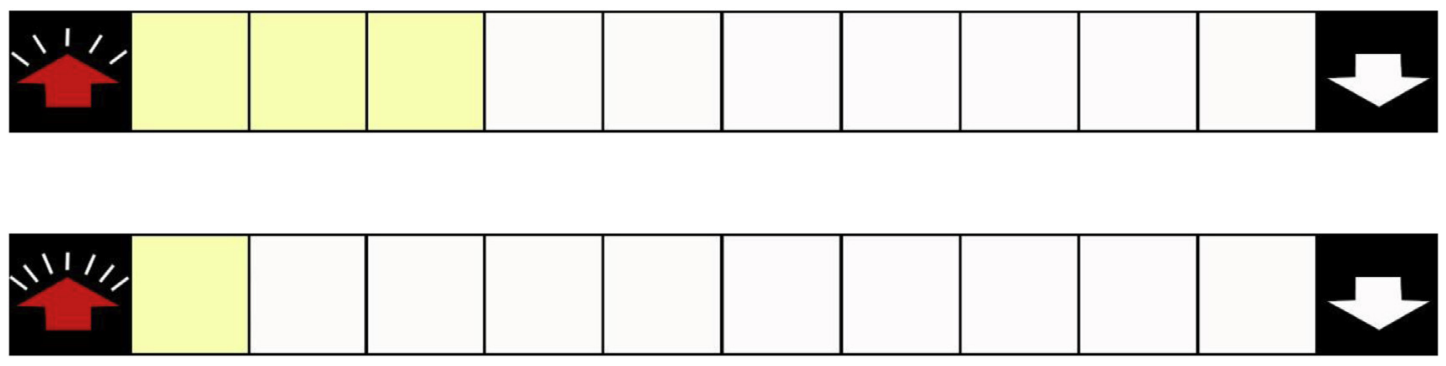
Version $\mathrm{C4}$

When the strength of the sun is too high the right arrow will turn red and flicker to indicate that the blinds need to go down.

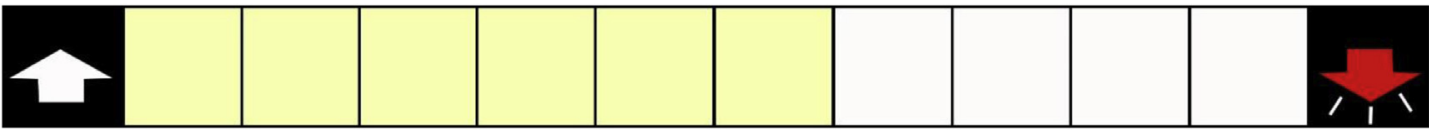

If you lower the blinds the arrow will turn off and stop flickering.

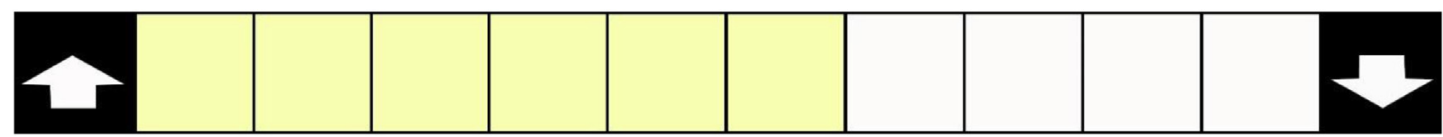

If you do not lower the blinds while the strength of the sun increases the arrow will gradually flicker faster.
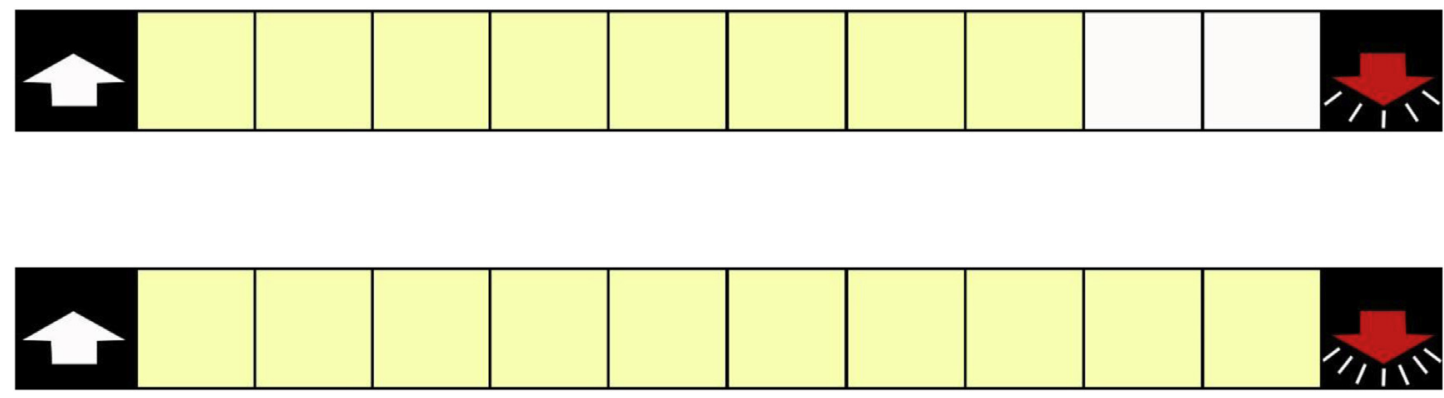

If you then still did not lowered the blinds they will automatically go fully down.

When the sun then loses strength again the left arrow will turn red and flicker to indicate that the blinds need to go up.

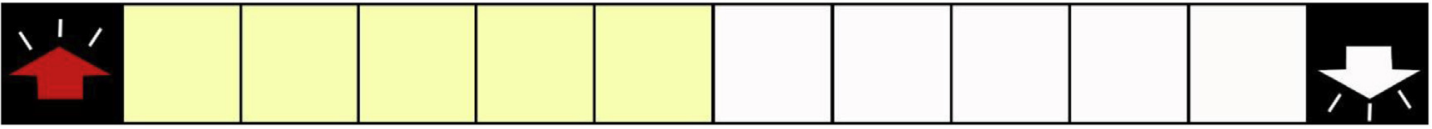

If you raise the blinds the arrow will turn off and stop flickering.

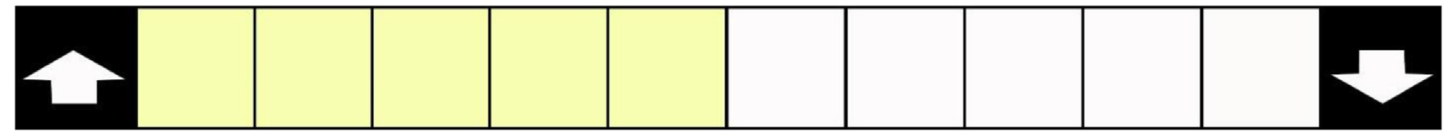

If you do not raise the blinds while the strength of the sun decreases the arrow will gradually flicker faster.

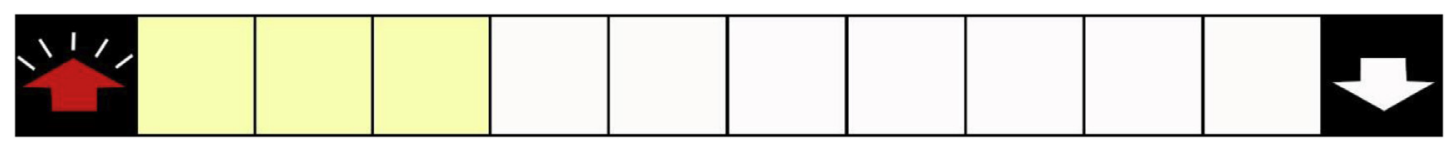

\begin{tabular}{|l|l|l|l|l|l|l|l|l|l|}
\hline N1/ & & & & & & & & & \\
\hline
\end{tabular}


If you then still did not raised the blinds they will automatically go fully down.

\section{Version CO}

This version has no light feedback system.

When the strength of the sun is too high the blinds will automatically go fully down.

When the sun loses strength again the blinds will automatically go fully up.

\section{Appendix B. Questionnaire experiment 1}

\section{Perceived system personality}

The statements below are about how you perceive the system to be. There are no wrong answers. Please indicate to which extent you think the statements apply to the system. $(0=$ not at all $6=$ completely).

\section{I find the blinds system...}

Reserved, Polite, Systematic, Relaxed, Creative, Talkative, Bold (= daring), Careless, Calm, Superficial, Energetic, Distant, Spontaneous, Easily discouraged, Curious, Withdrawn, Cooperative, Efficient, Moody, Liking routines

\section{Perceived control}

The statements below are about control. Please indicate to which extent you agree with them. $(0=$ strongly disagree $6=$ strongly agree)

1. I felt that I was in control of the blinds system

2. I was able to operate the blinds system in my own way.

\section{User satisfaction}

The questions below are about your satisfaction with the blind system. Please indicate to which extent you are satisfied. ( 0 = very dissatisfied $-6=$ very satisfied)

1. How satisfied are you with the overall blinds system?

2. How satisfied are you with the light feedback system?

3. How satisfied are you with the automatic blinds behaviour?

4. How satisfied are you with controlling the blinds system via the tablet?

\section{Appendix C. Questionnaire experiment 2}

\section{Perceived system personality}

The statements below are about how you perceive the system to be. There are no wrong answers. Please indicate to which extent you think the statements apply to the system. $(0=$ not at all $6=$ completely).

\section{1. I find the blinds system...}

Talkative, Cooperative, Reliable, Calm, Intelligent, Assertive, Friendly, Persistent, Relaxed, Curious, Helpful, Firm, Creative, Expressive, Polite, Consistent, Unemotional, Analytical, Confident, Agreeable, Patient, Preferring routine, Knowledgeable, Objective, Independent, Indecisive (hestitating), Impulse ridden, Dominant, Bossy, Timid

\section{Perceived control}

The statements below are about control. Please indicate to which extent you agree with them. $(0=$ strongly disagree $6=$ strongly agree)

1. I felt that I was in control of the blinds system

2. I was able to operate the blinds system in my own way.

\section{User satisfaction}

The questions below are about your satisfaction with the blind system. Please indicate to which extent you are satisfied. ( 0 = very dissatisfied $-6=$ very satisfied)

1. How satisfied are you with the overall blinds system?

2. How satisfied are you with the light feedback system?

3. How satisfied are you with the automatic blinds behaviour?

4. How satisfied are you with controlling the blinds system via the tablet?

\section{References}

[1] D. Aliakseyeu, B.W. Meerbeek, J. Mason, R. Magielse, S. Seitinger, Periphera interaction with light, in: S. Bakker, D. Housen, T. Selker (Eds.), Peripheral Interaction, MIT Press, 2016 (to appear in 2016).

[2] L.G. Bakker, E.C.M. Hoes-van Oeffelen, R.C.G.M. Loonen, J.L.M. Hensen, Use satisfaction and interaction with automated dynamic facades: a pilot study Build. Environ. 78 (2014) 44-52.

[3] Boeree, G. (2004), http://www.ship.edu/ cgboeree/bigfiveminitest.html, last visited 24-11-2015.

[4] R.J. Cole, Z. Brown, Reconciling human and automated intelligence in the provision of occupant comfort, Intell. Build. Int. 1 (1) (2009) 39-55.

[5] S. Escuyer, M. Fontoynont, Testing in situ of automatic ambient lighting plus manually controlled task lighting: office occupants' reactions, in: Proc. Lux Europa, 2001, pp. 70-75.

[6] A.D. Galasiu, J.A. Veitch, Occupant preferences and satisfaction with the luminous environment and control systems in daylit offices: a literature review, Energy Build. 38 (2006) 728-742.

[7] A. Guillemin, N. Morel, An innovative lighting controller integrated in a selfadaptive building control system, Energy Build. 33 (5) (2001) 477-487.

[8] H.B. Gunay, W. O'Brien, I. Beausoleil-Morrison, B. Huchuk, On adaptive occupant-learning window blind and lighting controls, Build. Res. Inf. 42 (6) (2014) 739-756.

[9] F. Haldi, D. Robinson, The impact of occupants' behaviour on building energy demand, J. Build. Perform. Simul. 4 (4) (2011) 323-338.

[10] P. Hinds, User Control and its Many Facets: a Study of Perceived Control in Human-computer Interaction (Technical report Hewlett-Packard), 1998.

[11] T. Inoue, T. Kawase, T. Ibamoto, S. Takakusa, Y. Matsuo, The development of an optimal control system for window shading devices based on investigations in office buildings, ASHRAE Trans. 94 (1988) 1049.

[12] M. Konstantoglou, A. Tsangrassoulis, Dynamic operation of daylighting and shading systems: a literature review, Renew. Sustain. Energy Rev. 60 (2016) $268-283$.

[13] A. Leaman, B. Bordass, Assessing building performance in use 4: the probe occupant surveys and their implications, Build. Res. Inf. 29 (2) (2001) 129-143.

[14] S.Y. Lee, J.L. Brand, Effects of control over office workspace on perceptions of the work environment and work outcomes, J. Environ. Psychol. 25 (3) (2005) 323-333.

[15] S. Maan, B. Merkus, J. Ham, C. Midden, Making it not too obvious: the effect of ambient light feedback on space heating energy consumption, Energy Effic. 4 (2) (2011) 175-183.

[16] R.A. Mangkuto, Modelling and Simulation of Virtual Natural Lighting Solutions in Buildings, Doctoral thesis, Eindhoven University of Technology, 2014

[17] R.A. Mangkuto, S. Wang, B.W. Meerbeek, M.B.C. Aries, E.J. van Loenen, Lighting performance and electrical energy consumption of a virtual window prototype, Appl. Energy 135 (2014) 261-273.

[18] R.R. McCrae, P.T. Costa, Validation of the five-factor model of personality across instruments and observers, J. Person. Soc. Psychol. 52 (1) (1987) 81.

[19] B.W. Meerbeek, M. Saerbeck, Designing domestic robots with personality, in: Kerstin Dautenhahn, Joe Saunders (Eds.), New Frontiers in Human-robo Interaction, vol. vi, 332, 2011, pp. 257-278.

[20] B.W. Meerbeek, E.J. van Loenen, M. te Kulve, M. Aarts, User experience of automated blinds in offices, in: Proceedings Experiencing Light 2012 (Int Conference on the Effects of Light on Wellbeing), Eindhoven, 2012.

[21] G. Newsham, J. Veitch, C. Arsenault, C. Duval, Effect of dimming control on office worker satisfaction and performance, in: IESNA Ann. Conf. Proc, 2004, pp. 19-41. 
[22] D. Norman, The Design of Future Things, Basic Books, 2007.

23] W. O'Brien, K. Kapsis, A.K. Athienitis, Manually-operated window shade patterns in office buildings: a critical review, Build. Environ. 60 (2013) 319-338.

[24] R. Parasuraman, Th Sheridan, A model for types and levels of human interaction with automation, IEEE Trans. Syst. Man Cybern. - Part A Syst. Humans 3 (2000) 286-297.

[25] Z. Pousman, J. Stasko, A taxonomy of ambient information systems: four patterns of design, in: AVI '06, 2006, pp. 67-74.

[26] M.S. Rea, Window blind occlusion: a pilot study, Build. Environ. 19 (2) (1984) 133-137.

[27] B. Reeves, C. Nass, The Media Equation: How People Treat Computers, Televisions, and New Media like Real People and Places, Cambridge University Press, New York, NY, 1996.

[28] C.F. Reinhart, K. Voss, Monitoring manual control of electric lighting and blinds, Light. Res. Technol. 35 (3) (2003) 243-260.

[29] S.A. Sadeghi, P. Karava, I. Konstantzos, A. Tzempelikos, Occupant interactions with shading and lighting systems using different control interfaces: a pilot field study, Build. Environ. 97 (2016) 177-195.

[30] E. Skinner, A guide to constructs of control, J. Person. Soc. Psychol. 3 (1996) 549-570.

[31] V. Venkatesh, M.G. Morris, G.B. Davis, F.D. Davis, User acceptance of information technology: toward a unified view, MIS Q. 27 (3) (2003) 425-478.

[32] J.A. Veitch, Psychological processes influencing lighting quality, J. Illum. Eng. Soc. 30 (1) (2001) 124-140.

[33] E. Vine, E. Lee, R. Clear, D. DiBartolomeo, S. Selkowitz, Office worker response to an automated venetian blind and electric lighting system: a pilot study, Energy Build. 28 (2) (1998) 205-218. 